\title{
Landscape of oncoplastic breast surgery across Poland
}

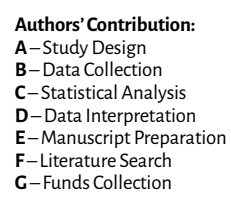

G-Funds Collection

\author{
Agnieszka Kołacinska ${ }^{1 A B C D E F}$, Diana Hodorowicz-Zaniewska ${ }^{2 B D E}$, Artur Bocian ${ }^{3 B D E}$, Dariusz \\ Michalik $^{4 \mathrm{BDE}}$, Rafal Matkowski ${ }^{\mathrm{BDE}}$, Andrzej Kurylcio ${ }^{6 \mathrm{BDE}}$, Paweł Pyka ${ }^{7 \mathrm{BDE}}$, Michał Charytonowicz $^{8 \mathrm{BDE}}$, \\ Maciej Berkan ${ }^{9 \mathrm{BDE}}$ \\ 'Dept of Head and Neck Cancer Surgery, Medical University of Lodz, Poland \\ ${ }^{2}$ Chair of Ceneral Surgery, Dept of Ceneral, Oncological and Gastroenterological Surgery, Jagiellonian University Medical College, \\ Cracow, Poland \\ ${ }^{3}$ Dept of Surgical Oncology, Holy Cross Cancer Center, Kielce, Poland \\ ${ }^{4}$ Dept of Ceneral Surgery, Ministry of the Interior and Administration Hospital, Olsztyn, Poland \\ ${ }_{5}^{5}$ Department of Oncology and Division of Surgical Oncology, Wroclaw Medical University; Breast Unit, Lower Silesia Oncology Center, Wroclaw, Poland \\ ${ }^{6}$ Dept of Surgical Oncology, Medical University, Lublin, Poland \\ 'Dept of Surgical Oncology, District Hospital, Walbrzych, Poland \\ ${ }^{8}$ Dept of Plastic Surgery, Center of Postgraduate Medical Education, Warsaw, Poland \\ ${ }^{9}$ Dept of Oncological Cynecology with Breast Surgery Division, Copernicus Memorial Hospital, Lodz, Poland
}

Article history: Received: 12.06.2017 Accepted: 30.10.2017 Published: 30.12.2017

ABSTRACT: $\quad$ Oncoplastic and reconstructive techniques are essential tools in the armamentarium of contemporary breast surgeons. The aim of the study was to identify oncoplastic reconstructive patterns in breast cancer centers across Poland. A questionnaire of 18 questions was sent by email to the members of the Polish Society of Surgical Oncology and the Polish Society of Plastic, Reconstructive and Esthetic Surgery via their dedicated websites. The numbers of breast cancer patients operated on in each center ranged from 120 to 904 per year. Breast conserving surgery (BCS) predominated in all but one center (range 50-70\%). Immediate breast reconstructions (IBR) accounted for 6-42\% of procedures, The most frequent type of IBR was either a two-stage expander followed by a permanent implant or one-stage implant-based with or without synthetic mesh. The most frequent type of delayed breast reconstruction (DBR) was a two-stage expander followed by implant- based reconstruction. None of the surveyed cancer centers performed free flap reconstruction. Deep inferior epigastric perforator (DIEP) flaps were performed in the plastic surgery department. Reconstructions based on pedicled flaps were performed in cancer centers. Acellular dermal matrices (ADM) and fat transfer were used in selected centers. In the clinical scenario of adjuvant radiotherapy, delayed breast reconstruction was favored. The full range of oncoplastic BCS was performed. Patient-reported outcome measures (PROM) and complications were assessed. Our findings can act as a platform for further improvement in skills, certification, data collection and audit, including patient reported expectation measures. There is also an urgent need to address pan-European inconsistencies in procedural reimbursement.

KEYWORDS: breast cancer, oncoplastic surgery, patient reported outcomes

\section{INTRODUCTION}

Oncoplastic and reconstructive techniques are essential tools in the armamentarium of contemporary breast surgeons. They are particularly useful in patients with larger or multifocal tumors who wish to preserve their breast by cosmesis, in patients with down-sized tumors following neoadjuvant chemotherapy, and in mastectomized patients who wish to have breast reconstruction [1-4]. Oncoplastic breast surgery was established in the 1990s and has been flourishing in recent years [5, 6]. However, many differences remain in the quality of breast cancer surgery across the various geographical areas of Europe [7]. In many countries there is still no formal training and structured curriculum in breast oncoplastic surgery [8]. Though many surgeons are technically able to do implants, this needs to be carried out within a multidisciplinary setting, to establish and agree on the appropriate treatment on a sound oncological basis [7]. Currently, data on Polish breast surgical practice in Poland is limited. There is a real need for benchmarking.

\section{AIM}

The aim of the study was to identify oncoplastic reconstructive patterns in breast cancer centers across Poland.

\section{MATERIALS AND METHODS}

A questionnaire of 18 questions was sent by email to the members of the Polish Society of Surgical Oncology and the Polish Society of Plastic, Reconstructive and Esthetic Surgery via their dedicated websites.

\section{RESULTS}

Breast surgeons from 7 of 14 cancer centers and 1 of 10 plastic surgery departments completed the questionnaire.

The numbers of breast cancer patients operated on in each center ranged from 120 to 904 per year. In all cancer centers a variety of oncoplastic/ reconstructive, immediate and delayed techniques were performed. In the single plastic surgery center that responded, only delayed reconstructions were performed, as it was not within the structure of a cancer institute. Of the seven cancer centers, oncoplastic or reconstructive techniques were performed solely by a surgical oncologist in five, and by a surgical oncologist or in collaboration with a plastic surgeon in two. In the single plastic surgery department, reconstructions were performed by a plastic surgeon independently. Breast conserving surgery (BCS) predominated in all but one center (range 50-70\%). A further center performed only $24 \%$ BCS.

Immediate breast reconstructions (IBR) accounted for $6-42 \%$ of procedures, depending on the center. The most frequent type of IBR was either a two-stage expander followed by a permanent implant or one-stage implant- based with or without synthetic mesh. 
The most frequent type of delayed breast reconstruction (DBR) was a two- stage expander followed by implant- based reconstruction.

None of the surveyed cancer centers performed free flap reconstruction. Deep inferior epigastric perforator (DIEP) flaps were performed in the plastic surgery department.

Reconstructions based on pedicled flaps, such as latissimus dorsi (LD) or transverse rectus abdominis muscle (TRAM) were performed in cancer centers. Acellular dermal matrices (ADM) were used in only one of the seven cancer centers. Fat harvesting and transfer was performed in 4 cancer centers and in the plastic surgery department.

In the clinical scenario of adjuvant radiotherapy planned after surgery, delayed breast reconstruction was favored or in very determined patients- immediate implant- based reconstruction, together with counselling about the risk of increased complications.

In 5 of 7 cancer centers, breast reconstructions were carried out after neoadjuvant chemotherapy. Patient-reported outcome measures (PROM) were assessed by a subjective scale, Breast Q questionnaire, photos or EORTC QoL in 5 of 7 centers. In 3 others such measures were planned to be implemented.

Complications after expander/ implant- based breast reconstructions occurred in 3-6\% patients (hematoma, infection, seroma, implant loss, extrusion, transposition). No data was available relating to capsular contracture.

In all cancer centers, the full range of oncoplastic BCS was performed (mammaplasties- vertical, inverted $\mathrm{T}$, dermoglandular flaps, round block, etc). According to surgeons participated in our study, availability of breast surgeons was insufficient in 4 of 7 cancer centers and in one of the plastic surgery departments.

Reimbursement of oncoplastic and reconstructive procedures by the National Health Fund was insufficient in all cancer and plastic surgery centers. Departments received 4843 Polish zloty for free flap breast reconstruction $(1$ euro $=4.4$ zloty), no reimbursement for adjusting contralateral breast symmetry, ADM and lipofilling, and there was no differentiation in reimbursement between classic BCS and the more sophisticated and time-consuming oncoplastic BCS. Reimbursement for bilateral procedures was inadequate. Data are shown in Table 1.

\section{DISCUSSION}

Breast surgeons need to deliver safe, affordable and timely surgery, including oncoplastic reshaping or reconstruction of the breast to all breast cancer patients [9]. This has a profound impact both on survival and femininity [10].

The majority of hospitals participating in this survey were high-volume centers treating at least 150 newly- diagnosed primary breast cancer patients, the minimum requirement for designation as a specialist breast center by the European Society of Breast Cancer Specialist (EUSOMA guidelines) [11]. Across Europe oncoplastic reconstruction is performed by specialist breast, general or plastic surgeons, sometimes in collaboration with each other; who should actually be involved may be under dispute and this needs to be addressed nationally [12].

This study shows that in the majority of centers surgical oncologists with oncoplastic skills carried out oncoplastic and reconstructive surgery. This might be due to some plastic surgery departments operating in isolation from cancer centers, thus limiting them to delayed reconstruction only. Surgical breast oncoplastic surgeons in Poland are now able to offer a broad spectrum of techniques, skills acquired by specialist training and attachments in high-profile international breast units and attendance at courses endorsed for example by the European Society of Surgical Oncology, and American or European Societies of Plastic Surgery.

In Poland, there is an urgent need for closer collaboration between surgical oncologists, general and plastic surgeons. A dedicated plastic surgeon with a special interest in breast reconstruction should be a part of every multidisciplinary team to ensure optimal initial evaluation of all breast cancer patients.

The most common type of IBR in Poland was found to be implantbased breast reconstruction. This figure is in line with UK figures given in the Second Annual Report of the National Mastectomy and Breast Reconstruction Audit (NMBRA). The prevalence of IBR in the UK was $38 \%$ in 2007 , rising to $54 \%$ in 2014 . The most frequent type of DBR procedure in the UK was free flap breast reconstruction, rising from $25 \%$ to $42 \%$ between 2007 and 2014 (range from $23 \%$ to $74 \%$ across region) [13]. In contrast, despite introduction of microsurgical flaps for breast reconstruction in Poland in 1996 (Witwicki et al.), the country lags behind. This has been attributed to the extensive learning curve in comparison with implant- based breast reconstruction and insufficient reimbursement by the National Health Fund [14].

In Poland approximately 50 free TRAM, DIEP and SIEA breast reconstructions are performed annually, and probably double that number in 2017 [15]. In contrast, in Germany, the annual number of free flaps for breast reconstruction is about 1200 , including 200 in Duesseldorf, where reimbursement is more generous (unilateral DIEP - 14,200 euro, bilateral DIEP- 18,500 euro, free TRAM- 9800 euro and pedicled TRAM- 7500 euro) (Andree, personal communication). A lack of standardized reimbursement for oncoplastic techniques across Europe, may well be one of the key reasons behind the difference in the number of free flap reconstructions between Western and Eastern Europe, which can be as great as 14 times.

The questionnaire also examined the optimum timing and form of breast reconstruction for the clinical scenario of postmastectomy radiotherapy. Cordeiro et al. prospectively found that the prevalence of severe capsular contracture grade 4 was 12 -fold higher in irradiated versus non-irradiated patients $(7.4 \%$ versus $0.6 \%$, respectively) and implant loss 13 -fold higher ( $9.1 \%$ versus $0.7 \%)$, but still $70 \%$ of irradiated women had good to excellent aesthetic results, and $95 \%$ would choose implants again [16]. Our findings indicate that when radiotherapy was planned, delayed reconstruction of the breast with autologous tissue e.g. LD plus implant, TRAM or DIEP if available, or fat transfer were performed and in determined patients implant- based breast reconstruction with disclosure of higher complication rates. In the latter cohort implant radiation was preferred by the majority of breast surgeons. This is in 
I IInI III

Minlte!

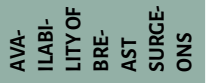

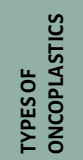

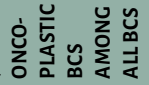

은

这崖

克

嵌吉

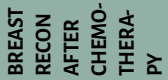

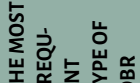

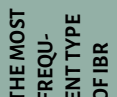

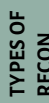

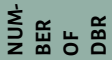

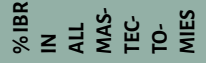

崖出安产

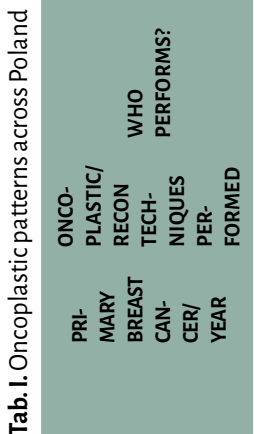

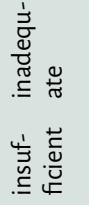

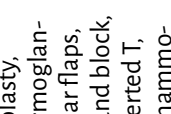

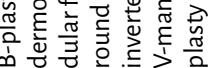

今

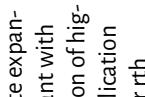

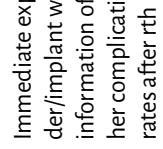

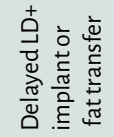

$\dot{\varepsilon}$

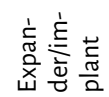

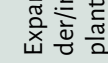

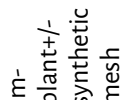

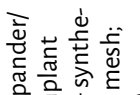

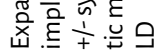

iे

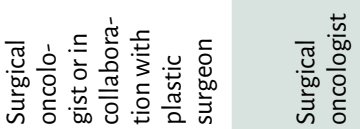

$\stackrel{n}{\wedge}$

ঃ

飑

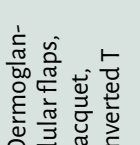

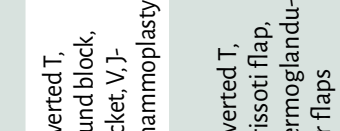

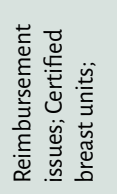

章:

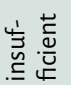

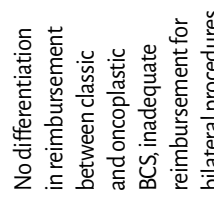

害

岁苞

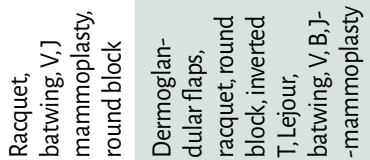

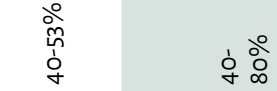

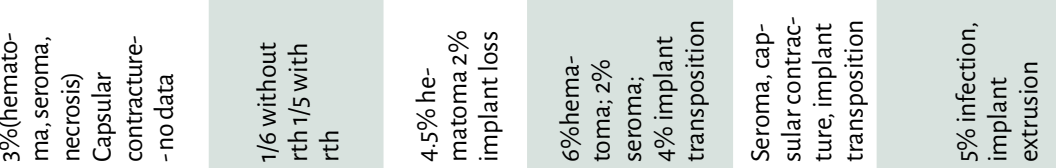

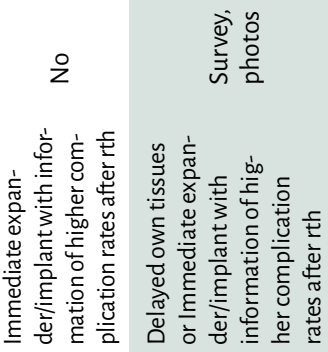

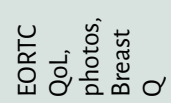

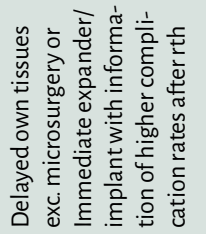

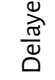

$\stackrel{\square}{\check{\nu}}$

ํㅜㄴ

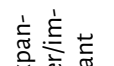

啨

部

$\stackrel{\check{\nu}}{\nu}$

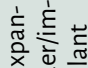

离

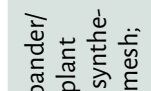

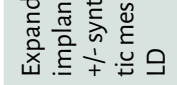

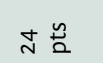

우욤ำ

$\stackrel{\infty}{\sim} \circ$

ㅇํ

ㅇํำ

$\stackrel{\circ}{\circ}$

今े

잉응

ㅇํํ

ঐ̊ำ

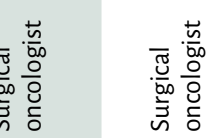

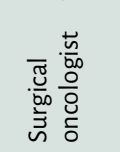

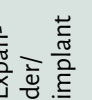

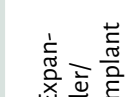

.

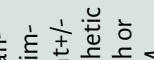

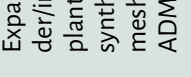

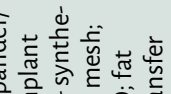

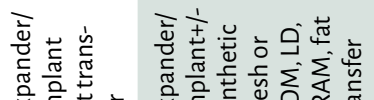

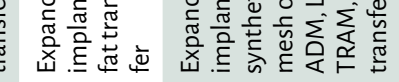

$\stackrel{\infty}{\wedge} \stackrel{\tilde{a}}{2}$

善

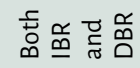

웅

ฟั

ㅇํำ

ถั่

䒽

กิ้

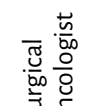

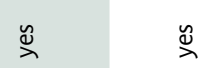

요욤

这
这童

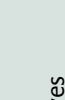

$\stackrel{\check{\varrho}}{\sim}$

斿

这

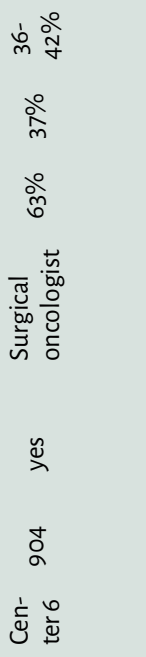

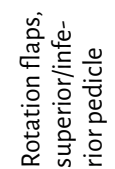

ํำ

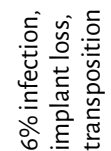

$\stackrel{\check{\nu}}{x}$

$\frac{\frac{n}{5}}{\frac{\pi}{2}}$

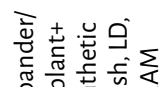

商.

항요욤

웅

ஓั่

品

ㅇํㅁ

㐫高

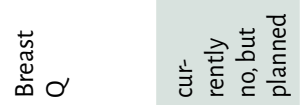

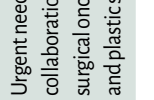

$\frac{\text { बे }}{\frac{0}{4}}$

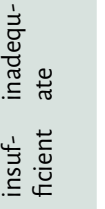

产

产

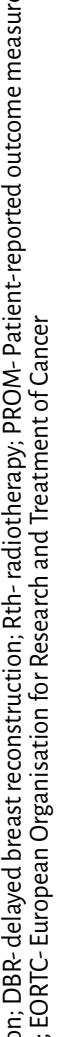

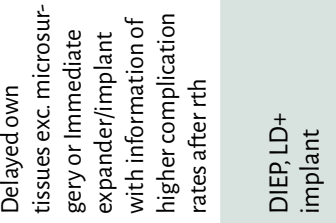

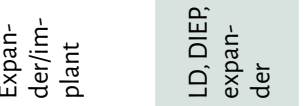
월

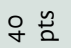

苍蛋

范

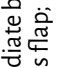

랄

흘

密㠃

它恼

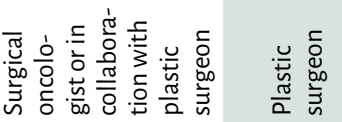

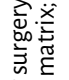

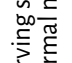

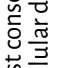

政
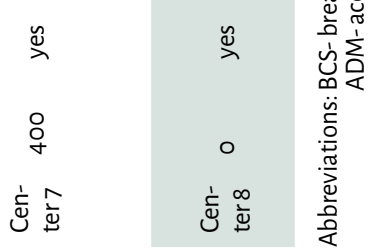
line with observations made by Cordeiro et al. that there was a 2 -fold increase in predicted failure rates for patients with tissue expander radiation than for patients with permanent implant [17]. Andree et al. described an intriguing IDEAL concept (Immediate implant DElayed AutoLogous) for patients in whom radio-chemotherapy is needed, either in a neoadjuvant or adjuvant setting [18]. Nipple- sparing mastectomy is performed by a breast surgeon from the senology department. If the patient opts for definitive autologous reconstruction, an immediate implant reconstruction is inserted epipectorally, followed by a DIEP flap performed 4-6 months later by a plastic surgeon with microsurgical skills. If a patient prefers a definitive implant- based reconstruction, this is carried out using a subpectoral approach with synthetic mesh or acellular dermal matrix. In capsular contracture or other complications arise, the implant is removed and replaced by a flap [18].

Complication rates (3-6\%) after implant-based breast reconstruction in Poland were comparable with institutions in Western Europe. In the UK, for example, the NMBRA database shows a 3\% complication rate (infection requiring antibiotics or removal, displacement requiring reposition) [19] and in Spain 5\% (infection, extrusion) [20].

The availability of oncoplastic and reconstructive techniques varies across Europe. This questionnaire has shown that in Poland there is an insufficient number of breast surgeons. In the UK in 2010 , immediate breast reconstruction rates varied significantly between 9\% and 43\% across the 30 English Cancer Network and this could not be explained by the socio-demographic and clinical characteristics of the women treated [19]. In Ukraine there were marked discrepancies between private and public institutions. In the private sector in Kiev, $52 \%$ of patients had immediate breast reconstruction, and the full range of oncoplastic techniques is offered [21].

In conclusion, there is a need for certified breast units and standardized oncoplastic techniques performed by specialist oncoplastic breast surgeons across Europe. Variation is more marked between level I and level II oncoplastic procedures and implant vs. flap- based breast reconstruction. However, there is an ongoing expansion in oncoplastic reconstruction, in terms of numbers performed, variety and complexity of techniques. For example there has been a 6-fold increase with low complication rates in some Eastern European countries over the last decade.

European breast surgeons are eager to improve their skills, as evidenced by fully or overbooked ESSO-endorsed breast surgery courses and other high quality workshops. Applications for the European Examination in Breast Surgery (EBSQBS) continue to increase.

Available data is inevitably biased due to the absence of a dedicated oncoplastic registry in Poland as in most European countries, incomplete or skewed data from individual surgeons or centers. However, our findings can act as a platform for further improvement in skills, certification, data collection and audit, including patient reported outcomes and expectation measures. There is also an urgent need to address pan-European inconsistencies in procedural reimbursement.

\section{REFERENCES:}

1. Mennie JC, Mohanna PN, O’Donoghue JM, Rainsbury R, Cromwell DA. National trends in immediate and delayed post-mastectomy reconstruction procedures in England: A seven-year population- based cohort study. Eur J Surg Oncol 2017; 43: 52-61.

2. Aggarwal S, Marla S, Nyanhongo D, Kotecha S, Nathbasu N. Current practice of therapeutic mammaplasty: a survey of oncoplastic breast surgeons in England. International Journal of Surgical Oncology 2016. doi: 10.1155/2016/1947876

3. Mansell J, Weiler-Mithoff E, Stallard S, Doughty JC, Mallon E, Romics L. Oncoplastic breast conservation surgery is oncologically safe when compared to wide local excision and mastectomy. Breast 2017; 32: 179-185.

4. Weber WP, Soysal SD, Fulco I, Barandun M, Babst D et al. Standarization of oncoplastic breast conserving surgery. Eur J Surg Oncol 2017. doi: 10.1016/j. ejso.2017.01.006.

5. Clough KB, Cuminet J, Fitoussi A, Nos C, Mosseri V. Cosmetic sequelae after conservative treatment for breast cancer: classification and results of surgical correction. Ann Plast Surg 1998; 41: 471-81.

6. Audretsch W, Rezai M, Kolotas C, Zamboglou N, Schnabel T, Bojar H. Tumour-specific immediate reconstruction in breast cancer patients. Persp Plast Surg 1998; 11: 71-100.

7. Wagstaff A. The invisible cure. Should we be talking more about cancer surgery? CancerWorld 2016; 73/73: 5-10.

8. Are C, Caniglia A, Malik M, Cummings C, Lecoq C, Berman R, Audisio R, Wyld L. Eur J Surg Oncol 2016; 42: 767-78.

9. Sullivan R, Alatise OI, Anderson BO, Audisio R, Autier P et al. Global cancer surgery: delivering safe, affordable, and timely cancer surgery. Lancet Oncol 2015; 11: 1193-224.

10. Pukancsik D, Kelemen P, Ujhelyi M, Kovacs E, Udvarhelyi N et al. Objective decision making between conventional and oncoplastic breast-conserving surgery or mastectomy: An aesthetic and functional prospective cohort study. Eur J Surg Oncol 2017; 43: 303-310.

11. Cardoso F, Cataliotti L, Costa A, Knox S, Marotti L, Rutgers E, Beishon M. European Breast Cancer Conference manifesto on breast centers/ units. Eur J Cancer 2017; 72: 244-250.

12. Carstensen L, Rose M, Bentzon N, Kroman NT. Knowledge and opinions on oncoplastic surgery among breast and plastic surgeons. Dan Med. J 2015; 62: 1-5.

13. Jeevan R, Cromwell DA, Browne JP, Caddy CM, Pereira J et al. Findings of a national comparative audit of mastectomy and breast reconstruction surgery in England. J Plast Reconstr Aesthet Surg 2014; 67: 1333-44.

14. Witwicki T, Towpik E, Mazur S, Sienko L, Jaworski J. Breast reconsctruction using free TRAM flap with microvascular anastomoses to internal mammary vessels: report of 2 cases. Nowotwory Journal of Oncology 2001; 5: 499-501.

15. Ulatowski L, Kaniewska A. The use of the DIEP flap in modern reconstructive surgery. Pol Przegl Chir 2015; 87: 472-81.

16. Cordeiro PG, Albornoz CR, McCormick B, Hu Q, Van Zee K. The impact of postmastectomy radiotherapy on two-stage implant breast reconstruction: an analysis of long-term surgical outcomes, aesthetic results, and satisfaction over 13 years. Plast Reconstr Surg 2014; 134:588-95.

17. Cordeiro PG, Albornoz CR, McCormick B, Hudis CA, Hu Q, Heerdt A, Matros E. What Is the Optimum Timing of Postmastectomy Radiotherapy in Two-Stage Prosthetic Reconstruction: Radiation to the Tissue Expander or Permanent Implant? Plast Reconstr Surg 2015; 135:1509-17.

18. Nestle-Krämling C, Bölke E, Budach W, Andree C. Breast reconstruction after neoadjuvant radio chemotherapy: review and personal technique IDEAL concept REV-EJMR-D-15-00268. Eur J Med Res 2016; 2: 24. doi: 10.1186/s40001016-0219-8. Review. Erratum in: Eur J Med Res. 2016;21:30.

19. http://www.content.digital.nhs.uk/catalogue/PUB02731/clin-audi-supp-prog-mast-brea-reco-2011-rep1.pdf

20. http://www.essoweb.org/eurecca-inspire/

21. http://www.ncru.inf.ua 


\begin{tabular}{llll}
\hline Word count: 2210 & Page count: 5 & Tables: 1 & Figures: -
\end{tabular}

DOl: $\quad 10.5604 / 01.3001 .0010 .6735$

Table of content: https://ppch.pl/resources/html/articlesList?issueld=10479

Copyright: Copyright @ 2017 Fundacja Polski Przegląd Chirurgiczny. Published by Index Copernicus Sp. z o. o. All rights reserved.

Competing interests: The authors declare that they have no competing interests.

2 The content of the journal „Polish Journal of Surgery" is circulated on the basis

of the Open Access which means free and limitless access to scientific data.

This material is available under the Creative Commons - Attribution 4.0 GB. The full terms of this license are available on: http://creativecommons.org/licenses/by-nc-sa/4.0/legalcode

Corresponding author: Agnieszka Kolacinska, MD, PhD, Dept of Head and Neck Cancer Surgery, Dept of Surgical Oncology, Medical University of Lodz, Cancer Center, Paderewskiego 4, 93-509 Lodz, Poland, Phone: +48 4268954 59, Email: agnieszka.kolacinska@umed.lodz.pl

Cite this article as: Kolacinska A., Hodorowicz-Zaniewska D., Bocian A., Michalik D., Matkowski R., Kurylcio A. , Pyka P., Charytonowicz M., Berkan M.; Landscape of oncoplastic breast surgery across Poland; Pol Przegl Chir 2017: 89 (6): 12 - 16 